\title{
« Mal tomber» : sur trois (mé)chances du mal chez Jacques Derrida
}

\author{
Nicholas Cotton \\ Université de Montréal
}

\begin{abstract}
Avoir mal, faire mal, vouloir du mal, en vouloir à quelqu'un: j'imagine déjà les souffrances du traducteur ou de la traductrice qui voudrait respecter chacun de ces trois mots, d'avoir à faire mal, sans parler de vouloir du mal à quelqu'un. Traduction apparemment impossible. La langue française me semble être la seule à faire un tel sort ou un tel accueil à la configuration inouïe et absolument singulière de ces mots, de ces très grands mots : avoir, faire, vouloir et mal.

Jacques Derrida, États d'âme de la psychanalyse
\end{abstract}

Le rapport entre "méchanceté » et "perversité » est depuis très longtemps tissé de retournements chiasmatiques et 
d'emprunts, d'ambiguïtés conceptuelles et historiques ${ }^{1}$. Se poser la grande question du mal ne peut se faire en effet sans un regard oblique vers celle de la perversité, qui la rejoue constamment. Les conceptions de la méchanceté sont à la fois riches et diverses, si bien que cette idée revêt différents visages, variant selon plusieurs facteurs (époque, champs de spécialisation, lexique, etc.). Nous avons tenté ailleurs une étude des multiples «visages » de la de perversité, non sans arriver à des conclusions similaires au sujet de cette autre idée tout aussi protéiforme ${ }^{2}$. Il n'est pas vain de rappeler d'entrée de jeu que ces quelques chassés-croisés historiques entre «méchanceté » et «perversité » mobilisent les notions d'ordre et d'intentionnalité. Le méchant serait précisément celui qui fait le mal en toute connaissance de cause, celui «qui fait délibérément du mal ou cherche à en faire, le plus souvent de façon ouverte et agressive" (Le Robert), alors que le pervers serait quant à lui celui qui a certes un « goût immodéré pour le mal» (Le Robert), mais qui serait aussi dépassé par une inclination impulsive. Comme le montre la psychanalyse, le pervers est non seulement celui qui a une disposition pour le

\footnotetext{
1 Le présent texte reprend, sous une forme retravaillée, deux communications, presque simultanées, données à l'automne 2015 : «"Mal tomber" : (mé)chance de la perversité chez Jacques Derrida», Colloque international "Visages et vicissitudes de la méchanceté», Association des étudiants des cycles supérieurs (AECS) du Département de français, Université d'Ottawa, septembre 2015, et « La méchance du mal : politique du code et de l'aléa », Journée d'étude «Jacques Derrida: politique et esthétique » organisée par Ginette Michaud et Georges Leroux à l'Université de Montréal en octobre 2015.

2 Voir " "Mettre sens dessus dessous" : repenser la perversité», dans Actes du colloque annuel de la Société des études supérieures du Département d'études françaises (Université de Toronto) 2012, <http:// french.chass.utoronto.ca/SESDEF/\#>, 2013.
} 
mal, mais aussi celui qui a besoin de la loi, spécifiquement dans le but de la transgresser ${ }^{3}$.

Cette réflexion éminemment complexe et toujours en cours n'a pas manqué de faire l'objet de plusieurs ouvrages de Jacques Derrida, qui s'est longuement intéressé à la question du mal, notamment dans ses Séminaires. Pour lui, il s'agit de déconstruire les notions d'intentionnalité et les oppositions un peu rigides entre les idées de mal, de méchanceté, de cruauté et de perversité. Dans ce texte, nous examinerons de quelles manières il est possible de repenser ces idées à l'aide du concept de "méchance», que Derrida introduit à l'occasion dans ses textes pour inquiéter ces notions et leurs représentations traditionnelles. Pour ce faire, nous aborderons d'abord la question de la méchance de manière à la comprendre abstraitement et théoriquement. Nous nous intéresserons ensuite, toujours à partir du corpus derridien, à trois formes particulières que prend celle-ci : les méchances pragmatique, politique et radicale. Le tout devrait permettre d'enrichir notre réflexion sur la méchanceté et d'en étoffer notre compréhension.

\section{La «méchance insignifiante du mal»}

«Méchance» est un terme étrange, hybride et polysémique, qu'en fin de compte Derrida n'utilise pas lui-même très fréquemment. Il lui préfère bien souvent «méchanceté » ou «malchance», mais il arrive à l'occasion qu'il opère la conjonction des deux mots pour en créer un nouveau. C'est le cas en particulier à la toute fin d'États d'âme de la psychanalyse,

\footnotetext{
3 À ce sujet, il faudrait notamment relire Henri Rey-Flaud (2002, 2014), mais aussi Aulagnier (1981), Castel (2014), Stoller (1978).
} 
où il parle d'une "méchance insignifiante du mal » (p. 90), et encore dans L'animal que donc je suis, où il en donne cette fois une définition bien précise :

Il se passe là quelque chose qui ne devrait pas avoir lieu comme tout ce qui arrive, en somme, un lapsus, une chute, une défaillance, une faute, un symptôme (et symptôme, vous le savez, cela veut dire aussi la chute: le cas, l'événement malheureux, la coïncidence, l'échéance, la méchance). (p. 19)

La combinaison des termes «méchanceté » et «malchance » n'est donc pas uniquement lexicale ou réductible à un simple jeu de mots, elle est également sémantique. La défaillance peut être une " chance » pour Derrida, qui, dans un texte intitulé «Mes chances. Au rendez-vous de quelques stéréophonies épicuriennes », associe encore la malchance à un mouvement vertical de chute. Le titre de ce texte est d'ailleurs tout à fait significatif puisque l'on entend bien l'homophonie signifiante entre "Mes chances», un déterminant possessif suivi du vocable "chance» au pluriel, et "Méchance», néologisme qui signifierait le caractère général du méchant ou du malchanceux sans que ce ne soit tout à fait décidable. Dans ce texte qui porte en premier lieu sur une déconstruction de la notion de chance, Derrida précise que la "malchance est méchance» (1998, p. 358 ; Derrida souligne). En suggérant cela, le philosophe revient à notre toute première compréhension du mot «méchant». Les termes "méchant» et "méchanceté » dérivent tous les deux, en effet, du verbe désuet mescheoir, qui signifiait déjà en ancien français "mal choir», "qui tombe mal » (Rey, 1998, II, p. 2173) et non qui fait le mal. Il faudrait donc distinguer la méchance de la cruauté - un thème central du Séminaire La bête et le souverain, sur lequel il y aurait encore beaucoup à dire - , qui suppose le calcul, la souffrance, la 
blessure, la mutilation et, surtout, tout un plaisir « à souffrir ou à faire souffrir, voire à se faire souffrir » (Derrida, 2001a, p. 74).

Le tour de force de Derrida, ou encore le tour supplémentaire qu'il fait faire à cette idée, est de transformer cette malchance en chance. Bien sûr, le philosophe n'est pas superstitieux et il le montre par une longue lecture du chapitre "Déterminisme, croyance au hasard et superstition" de Psychopathologie de la vie quotidienne (Freud, 1967) dans « Mes chances ». Toutefois, il rend sensible cette double acception du mot "chance», qui peut vouloir dire tour à tour hasard, probabilité et éventualité heureuse. Comme le lapsus, propose Derrida, "la méchance inverserait son signe, elle serait la chance pour la vérité de se révéler [...] c'est peut-être cette possibilité pour mes chances d'être une malchance et pour ma méchance d'être en vérité une chance» $(1998$, p. 374 ; nous soulignons). Elle est une chance parce qu'elle ouvre un espace incertain de possibilités pour permettre à l'événement digne de ce nom - c'est-à-dire toujours radicalement imprévisible d'arriver. Rappelons que, pour Derrida, un événement ne peut jamais en être un que s'il est imprévisible, sans quoi il n'est pas un "événement» au sens propre. Il l'explique d'ailleurs très bien dans Dire l'événement, est-ce possible?, où il reprend les motifs de la chute et de la verticalité :

L'arrivée de l'arrivant c'est l'autre absolu qui tombe sur moi. J'insiste sur la verticalité de la chose, parce que la surprise ne peut venir que d'en haut. [...] l'événement en tant qu'événement, en tant que surprise absolue, doit me tomber dessus. Pourquoi? Parce que s'il ne me tombe pas dessus, cela veut dire que je le vois venir, qu'il y a un horizon d'attente. (p. 97) 
Le sens de cette chute, de ce «mal tomber», n'est pensable que du point de vue très fini de l'homme et non de celui d'une dissémination constituée de renvois sans origine. Elle est impensable du point de vue de ce que Derrida appelle tantôt une «citationnalité générale » (1990, p. 45), tantôt une « pervertibilité structurelle » (2004a, p. 189) de toute chose. Là, au contraire, la chance n'est jamais une « chance ». La méchance est donc ce moment ou plutôt cet indice de la chute et de l'échec qui entraîne avec elle la possibilité nouvelle d'un rapport renouvelé au monde et à tout ce qui se constitue en programme ou en code. Une "chance» à saisir en quelque sorte. Par conséquent, il ne faudrait pas en déduire que cette condition est tragique. En s'appuyant sur une conception de la langue où les mots «contiennent leur propre perversité » (Derrida, 2013, p. 35), la méchance peut agir à titre de relance. C'est ce que nous observerons à partir de maintenant.

\section{Pragmatique du performatif : quelque chose qui tourne mal}

Pour Jacques Derrida, les mots ont en eux-mêmes une réserve de perversité, à savoir la possibilité toujours présente et menaçante de ne pas arriver à destination ou de mal y arriver, de mal tomber ou de mal tourner. Suivant la pensée derridienne, il est possible de dire que l'ambiguïté significative de termes comme « méchanceté » et « perversité » dans le langage courant - dont on pourrait montrer l'instabilité dénotative et connotative, historique et sociale - est aussi en quelque sorte, et heureusement, leur chance. S'il y a quelque chose sur lequel la lecture des textes derridiens nous instruit, c'est bien qu'il faille continuellement déjouer les équivoques et les faire raisonner entre elles, les rejouer, l'une contre l'autre. Ne seraitce pas d'ailleurs un sens possible de la déconstruction? Ne pas 
avoir peur de tenter sa chance ou de courir le risque - «prendre la chance», comme on dit au Québec - d'entretenir une possibilité de menace, ce que Derrida appellerait peut-être la «méchance».

Dans un débat bien connu des années 1970 autour des énoncés performatifs et de la théorie des actes de langage d'Austin, Jacques Derrida et John Searle se sont mesurés l'un à l'autre autour d'une compréhension plutôt différente du langage et de la communication. Pour Derrida, dans un texte intitulé «Signature Événement Contexte», il est clair que l'énoncé performatif est itérable, tout comme chaque mot qui le compose. Concrètement, le mot est une marque et se caractérise de ce fait par sa rupture, ou par la possibilité d'une telle rupture, par rapport à un contexte précis (la marque peut toujours être démarquée ou remarquée). Le paradoxe est le suivant : pour être une marque, cette dernière doit avoir la possibilité d'être marquée de nouveau - répétée - mais c'est aussi ce qui l'empêche d'être absolument signifiante. Elle est toujours prise dans un mouvement de répétitions et de réappropriation. Le mot comme marque n'est dès lors jamais tout à fait identique à luimême parce que, justement, il peut performer dans une multitude de contextes. Pour Derrida, un énoncé performatif manque donc toujours un peu ce qu'il fait. Or, cette condition que l'on pourrait associer à une malchance, à un malheur ${ }^{4}$, ou encore à une "méchante conséquence » (Derrida, 2000, p. 89), est aussi une chance : «Cela imprime au mouvement même [de la marque] un pouvoir de détournement [...]. Pas de destination assurée:

\footnotetext{
${ }^{4}$ Austin appelle performatif malheureux [infelicity] le performatif qui n'est pas réalisé en contexte adéquat.
} 
précisément parce qu'il y a de la marque [...] autrement dit de l'insignifiance. » (Derrida, 1998, p. 369 ; Derrida souligne)

Prenons l'exemple d'un performatif en particulier: la promesse. Advenant le cas où elles respectent un certain cadre conventionnel, les promesses font advenir leur propre réalité au moment où elles sont prononcées. Sur ce point, Austin, Searle et Derrida pourraient être d'accord. Il faut se rappeler cependant, avec Derrida, que « [s]i la promesse était automatiquement tenue, ce serait une machine, un ordinateur, un calcul » (Derrida, 2001b, p. 109) et non une promesse. Ainsi, la menace potentielle qui la guette, celle de l'échec, est également ce qui lui garantit une existence effective. Dans La Philosophie au risque de la promesse, Derrida précise :

tout en respectant la légitimité, tout à fait indispensable, de l'analyse des speech acts qui veut que la promesse ne soit jamais une menace, tout en respectant les analyses herméneutiques et phénoménologiques qui se règlent sur cette axiomatique-là, je me demande [...] si il n'y aurait pas une [...] pervertibilité structurelle qui fait que le don se retourne en poison, ou bien que, en obligeant autrui à entrer dans le cadre de la promesse, je fasse du mal en faisant ce qui me paraît le bien, je produise quelque chose qui tourne mal ; autrement dit que le méfait soit au travail dans le bienfait ou, plus exactement, que la possibilité du méfait soit au travail dans le bienfait. (p. 197 ; Derrida souligne)

Dans ce texte, Derrida va donc plus loin en admettant qu'il puisse y avoir une "pervertibilité structurelle» et que cette dernière est inexorablement liée au mal, que ce soit au mal faire (méfait) ou à la mauvaise fortune (mal tourner). L'itérabilité, nous dit Derrida dans « Mes chances », c'est donc aussi et surtout « un phénomène de chance ou de hasard, une infelicity comme on dit parfois dans la théorie austinienne [...] pour désigner des 
déviations accidentelles ou parasitaires dans la production des performatifs, des promesses, des ordres ou des serments » (1998, p. 358).

\section{Politique : le code et l'aléa}

Derrida a aussi plusieurs fois associé la question du mal à celle du politique. Dans le Séminaire La Bête et le souverain, par exemple, il tente de déconstruire une certaine conception classique et dominante de la souveraineté des États-nations qui vient traditionnellement avec l'idée que le politique suppose «l'ennemi, la possibilité de la guerre, la méchanceté de l'homme» (Derrida, 2008, p. 111). La parution au printemps 2015 de la transcription d'un débat (2003) avec Jean Baudrillard témoigne en outre de l'actualité de la pensée politique de Jacques Derrida. Dès le départ, le psychanalyste René Major, qui anime et présente ce débat, a voulu l'inscrire à la suite de la célèbre correspondance entre Freud et Einstein

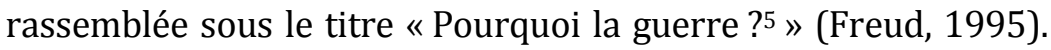
Il est tout à fait significatif que l'ouvrage de 2015 ait pour sujet la guerre et le terrorisme. Plus précisément, la controverse porte sur l'événementialité des «événements » du 11 septembre et sur l'opposition entre pulsion de cruauté et pulsion de mort. Comment concilier effectivement l'exigence derridienne et hyperbolique du concept d'événement - radicalement imprévisible - et la réalité d'un événement particulier comme celui du 11 septembre? René Major demande en ce sens à Derrida de préciser sa pensée sur ce que serait une « raison qui tient compte de l'inconscient [et] qui transige [...] "entre d'un

5 Corpus auquel s'était déjà confronté Derrida dans États d'âme de la psychanalyse (2000). 
côté l'exigence raisonnée du calcul et de la conditionnalité et, de l'autre côté, l'exigence intransigeante, c'est-à-dire non négociable, de l'incalculable inconditionnel6" " (Derrida, 2015, p.34). Non seulement penser d'une part le code et l'«aléa de rencontre» (Derrida, 2000, p. 90) d'autre part, mais encore penser ensemble le code et l'aléa.

Pour l'un, Baudrillard, le 11 septembre est précisément un événement - survenu, advenu - duquel l'intervention en Irak découle, mais sans que cette conséquence soit nécessaire. Pour l'autre, Derrida, le 11 septembre n'est pas un événement à proprement parler; il n'est pas à l'origine, du moins, de l'invasion américaine éventuelle en Irak, dont les prémisses précèdent 2001 et les fameux événements ${ }^{7}$. Derrida va encore plus loin dans ce débat; cet épisode ouvre du point de vue de la souveraineté la possibilité pour demain d'une menace - d'un mal - bien pire pour l'État américain. Toute la question est de savoir si ce mal pourrait être une chance, une sorte de méchance du politique. Derrida ne répond pas directement à la question posée par Major dans Pourquoi la guerre aujourd'hui ? comme nous serions en droit de nous y attendre, mais cela ne veut pas dire qu'il ne l'a pas déjà fait ailleurs, dans Mes chances » par exemple, où il écrit que

[1]a langue n'est que l'un de ces systèmes de marques qui ont tous pour propriété cette étrange tendance: accroître simultanément les réserves d'indétermination aléatoire et les

\footnotetext{
${ }^{6}$ Le passage entre guillemet est de Derrida, cité par R. Major. Cette question est une reformulation de celle que l'on retrouve sous la plume de Derrida dans un autre texte : « Comment penser ensemble la machine et l'événement, une répétition machinale et ce qui arrive?» (Derrida, 2001a, p. 74 ; Derrida souligne)

7 Sur ce point, René Major notait en 2014 que l'Histoire aura donné raison à Derrida. Voir « Actualisation » (Derrida, 2015, p. 67-85).
} 
pouvoirs de codage ou de surcodage, autrement dit de contrôle et d'autorégulation. Cette concurrence entre l'aléa et le code perturbe la systématicité même du système dont elle règle pourtant le jeu dans son instabilité. (Derrida, 1998, p. 355 ; Derrida souligne)

Il est clair que le système politique, ou plutôt le système $d u$ politique, est un exemple de cette « loi de déstabilisation » (Derrida, 1998, p. 355).

L'essentiel de la controverse entre Baudrillard et Derrida reposait sur cette idée d'une démocratie ouverte et imprévisible («à-venir» dans les mots de Derrida). Pour Baudrillard, non seulement Derrida minimise l'impact symbolique et réel du 11 septembre, mais il s'illusionne sur une supposée prise de conscience collective qui prendrait la forme d'une nouvelle raison politique. Il n'y a que l'événement du 11 septembre qui puisse répondre symboliquement à la surpuissance occidentale, selon l'auteur de L'Esprit $d u$ terrorisme (2002). Or cette réponse annonce quant à elle une ère noire de la démocratie; elle inaugure plutôt une mondialisation du pouvoir, de la puissance et de la souveraineté occidentale. Pour Derrida, qui précise explicitement qu'il se considère au contraire "sans illusion» et "sans le moindre optimisme» (2015, p.56), cette guerre est toutefois une prétendue guerre et ces terroristes de prétendus terroristes. L'« événement » du 11 septembre n'est pas un événement, car il était tout à fait prévisible. Dans un texte consacré au même sujet, Derrida rappelle qu'un « événement c'est ce qui arrive et en arrivant arrive à me surprendre, à surprendre et à suspendre la compréhension, l'événement c'est d'abord ce que d'abord je ne comprends pas. Mieux, l'événement, c'est d'abord que je ne comprenne pas. » (Derrida, 2004b, p. 139 ; Derrida 
souligne) L'événement se trouve dès lors plutôt du côté de ce qui échoue, de la méchance, à savoir là où il n'y a pas précisément eu événement, mais où entre le code et l'aléa il y aurait une sorte de glitch :

Bien que l'expérience d'un événement, le mode selon lequel il nous affecte, appelle un mouvement d'appropriation (compréhension, reconnaissance, identification, description, détermination, interprétation à partir d'un horizon d'anticipation, savoir, nomination, etc.), bien que ce mouvement d'appropriation soit irréductible et inévitable, il n'y a d'événement digne de ce nom que là où cette appropriation échoue sur une frontière. (Derrida, 2004b, p. 139; Derrida souligne)

Derrida semble dire, dans Pourquoi la guerre aujourd'hui?, que si l'on peut parler d'événement, ce serait plutôt dans «le fait inouï malgré tout [...] que les États-Unis avant de déclencher la guerre [en Irak] à laquelle ils se préparent, [...] manifestent le besoin d'être approuvés, légitimés, cautionnés par une instance du droit international » (Derrida, 2015, p.57). Pour Derrida, il s'agit là d'un signe incontestable laissant entrevoir un changement qui serait radical parce qu'impensable, c'est-à-dire une nouvelle conception de la souveraineté. Sans s'abuser sur la réalité d'un tel changement, Derrida remarque, dans le sillage de Kant, que le signe d'une tentative derrière l'échec est aussi un signe de l'événement. Il observe en ce sens qu'au sujet de la Révolution française, « Kant disait que le fait que l'on a tenté de est un signe à la fois prometteur, commémorateur, et que ce signe est [en lui-même] un événement» (Derrida, 2015, p.56; Derrida souligne). Il s'agit ici précisément de ce que nous appelons la méchance, entendue comme malchance ou comme effet positivement pervers qui annonce quelque chose comme un $\grave{a}-$ 
venir meilleur: «En un autre temps les États-Unis auraient déclenché leur guerre sans demander l'avis de personne.» (Derrida, 2015, p. 57)

Cet avenir meilleur ne serait pas bêtement meilleur, mais meilleur au sens où il prend en charge "l'être politique du politique» (p.134), dit encore Derrida dans le texte «De l'hostilité absolue ». Tout se passe effectivement comme si, dans une logique auto-immunitaire, la communauté américaine mettait à mal son propre concept de démocratie au nom de cette même démocratie. C'est précisément ce qui ouvre une brèche, un aléa, une nouvelle ère possible mais non effective de la démocratie. "Ce qui est effrayant», constatait Derrida dans son Séminaire, "c'est l'hypocrisie d'un impérialisme qui se donne l'alibi de l'humanitaire universel (donc au-delà de la souveraineté d'un État-nation) pour, en fait, protéger ou étendre les pouvoirs d'un État-nation particulier. » (2008, p. 111 ; Derrida souligne) Cette frayeur, c'est par méchance ce qui ramène aussi la souveraineté à ce qu'elle est : un fantasme, une illusion. Du coup, cette méchance donne une chance au politique; elle s'avère encore une fois une «chance pour la vérité de se révéler » (Derrida, 1998, p. 374) qui pourrait bien à terme servir une « re-politisation » ne retombant pas « dans les mêmes ornières d'une "fiction malhonnête" » (Derrida, 2008, p. 112).

\section{Le mal : de l'altérité radicale}

Une troisième et dernière forme de méchance, celle-là peut-être encore plus fondamentale, se trouverait du côté du mal luimême. Serge Margel observe, dans un texte où il s'intéresse au mal radical chez Derrida, que depuis Foi et savoir, le philosophe 
réfléchit à cette question en termes d'immunité et d'autoimmunité. La religion et la raison, par exemple, pourraient bien venir d'une seule et même source selon Derrida, à savoir l'exigence de répondre de l'autre et devant l'autre. Or répondre devant l'autre, ce serait précisément restaurer ce que cette réponse menace d'un même geste, à savoir l'immunité, l'indemnité d'une altérité. En préservant la «pure » altérité de l'autre, c'est l'indemnité du propre qui est menacée et qui flanche. Cette exigence de répondre devant l'autre est aussi pour Derrida une responsabilité et elle se retrouve également dans des questions toutes concrètes comme celle de la traduction (que ce soit d'une langue à l'autre ou à l'intérieur d'une même langue comme nous invite à le penser le philosophe dans Le Monolinguisme de l'autre). Marc Crépon parle d'un paradoxe de l'intraductibilité dans Langue sans demeure, l'altérité radicale de l'idiome de l'autre appelle une traduction qui n'est rien de moins qu'une réduction répétée au même, au connu, au lisible. Crépon, avec Derrida, invite dès lors à la fois à la vigilance et au risque : «L'altérité de ce qui vient à moi, c'est cela qui sollicite ou même réclame ma traduction mais qui exige aussi, en même temps, d'être protégé, qui veut donc et ne veut pas être traduit. » (Crépon, 2005, p. 60 ; Crépon souligne)

Avant même les questions essentielles des langues et du politique, ce rapport d'altérité et de différences - il faudrait sans doute dire de différance - est sans contredit ce qui anime et rend problématique le lien communautaire. Margel propose dans son texte de considérer deux types de liens sociaux, un lien politique (institué) et un lien de pures singularités (primordial) «qui "précède" toute institution politique, mais qui se constitue ou se reconstitue en politique» (2011, p. 73). 
Postuler qu'il y ait un lien antérieur au lien politique comme le fait Derrida est très dangereux, ajoute-t-il, mais ce « désert de pure altérité ${ }^{\prime}$ (Margel, 2011, p. 74) constitue à la fois une chance et une menace, à savoir une méchance. Dans une logique bien marquée et peut-être inversée de l'auto-immunité, le mal radical serait cette dépendance à la menace. De son côté, J. Hillis Miller note, dans un texte paru récemment en français dans le collectif Appels de Jacques Derrida, qu'une des « dernières idées frappantes » de Derrida est celle que «toute communauté est affligée d'un désordre auto-destructeur » (2014, p. 261). Une autre «méchante conséquence», dirait peut-être Derrida. Miller fait un pas de plus par rapport à Margel en prenant toute la mesure d'un tel postulat. Dans ce texte qui tourne autour de la question du religieux à l'instar de celui de Margel, Miller montre effectivement que Derrida, contre tout réel optimisme, affirme qu'il n'y a pas de monde commun :

Derrida, de façon presque unique parmi les philosophes et théoriciens modernes, affirme l'isolement fondamental et irrémédiable de tout Dasein. Pour Derrida, [...] nul pont [...] nul communication ni aucun transfert ne relie ou ne peut jamais relier un moi insularisé à d'autres soi. [...] En corollaire, toute communauté est une construction artificielle et déconstructible fabriquée de mots ou d'autres soi. (Miller, 2014, p. 276)

Ce faisant, au milieu de ce qu'il serait possible d'identifier comme le cœur d'un pessimisme derridien, Miller rappelle que si Derrida refuse par-dessus tout d'appartenir à une famille ou à une communauté, c'est exactement parce que c'est dans

\footnotetext{
${ }^{8}$ La comparaison au « désert » vient de Derrida dans Foi et savoir.
} 
l'isolement - ultime méchance - que peut paradoxalement surgir un rapport éthique et responsable à l'autre ${ }^{9}$.

On le voit bien, si elles s'accrochent accidentellement et spectaculairement à l'actualité, les questions du mal, de la méchanceté et de la perversité traversent de bout en bout la pensée de Derrida. Ces notions, qui supposent toutes une organisation particulière de la morale et qui se font chaque fois le reflet d'une communauté de pensée et d'une époque, sont à déconstruire. En proposant d'explorer ces idées bien sédimentées à l'aide du concept opératoire de méchance, Derrida offre une réflexion risquée mais essentielle qui se produit dans les oscillations axiologiques, entre le bon et le mauvais ou le bien et le mal par exemple, et non dans le franchissement ou la prise de position. Rapprochement surprenant de la méchanceté et de la chance, la méchance est une chance dans le mal et l'échec. La chute, la déviation, le fait de «mal tomber» ou de "mal tourner» ne doivent plus être compris comme une inclination (ou une inclinaison) à sens unique et irrémédiable, mais comme une relance: le mal est une chance, un aléa imprévisible, un risque à prendre. Dans l'oscillation entre le code et l'aléa, la possibilité d'une ouverture heureuse à ce qui excède le programme.

Ce qui a été abordé ici, on le comprendra, n'est que le début d'une exploration qui pourrait être beaucoup plus sérieuse et qui pourrait toucher plus résolument l'espace littéraire. L'analyse derridienne de «La fausse monnaie» dans Donner le temps, pour ne nommer que cet exemple, est tout

${ }^{9}$ Miller cite $A$ taste for the Secret (2001) de Derrida. 
entière consacrée à une lecture des abîmes de méchance rendus possibles par le petit poème en prose de Baudelaire, si bien qu'avec le poète des Fleurs du mal, «faire le mal » peut devenir, dans un tour de "vices» supplémentaire, «mal faire le mal». Rappelons-nous ce personnage, nous met en demeure Derrida, et sa "perversion bête » qui consiste à ne pas faire «ce qu'il devait pour savoir qu'il était méchant, pour le faire savoir et pour se l'avouer» (Derrida, 1991, p. 213 ; Derrida souligne). Il faudra rendre compte plus exhaustivement un jour du potentiel et de l'omniprésence de cette idée de méchance dans le corpus derridien. Toute une étude pourrait y être consacrée : ce serait un risque et une chance.

\section{Bibliographie}

Aulagnier, Piera, Jean Clavreul et al. (1981), Le Désir et la perversion, Paris, Seuil, coll. « Points Essais ».

CASTEL, Pierre-Henri. (2014), Pervers. Analyse d'un concept suivi de Sade à Rome, Montreuil-sous-Bois, Ithaque, coll. «Philosophie, Anthropologie, psychologie».

CRÉPON, Marc. (2005), Langues sans demeure, Paris, Galilée, coll. « La philosophie en effet ».

DERRIDA, Jacques. (1990 [1971]), «Signature Événement Contexte ", dans Limited Inc, présentation et traduction d'Élisabeth Weber, Paris, Galilée, coll. «La philosophie en effet », p. 17-51.

-. (1991), Donner le temps I. La fausse monnaie, Paris, Galilée, coll. « La philosophie en effet ». 
-. (1994), Politiques de l'amitié suivi de L'Oreille de Heidegger, Paris, Galilée, coll. « La philosophie en effet ».

-. (1996a), Le Monolinguisme de l'autre ou La prothèse d'origine, Paris, Galilée, coll. « Incises ».

-. (1996b), Foi et savoir suivi de Le Siècle et le pardon, Paris, Seuil, coll. « Points Essais ».

—. (1998 [1987]), «Mes chances. Au rendez-vous de quelques stéréophonies épicuriennes », dans Psyché I. Inventions de l'autre, Paris, Galilée, coll. « La philosophie en effet », p. 353-384.

—. (2000), États d'âme de la psychanalyse. L'impossible au-delà d'une souveraine cruauté, Paris, Galilée, coll. « Incises ».

- (2001a), Papier Machine. Le ruban de machine à écrire et autres textes, Paris, Galilée, coll. « La philosophie en effet ».

- avec Gad SousSANA et Alexis Nouss. (2001b), Dire l'événement, est-ce possible?, Paris, L'Harmattan, coll. «Esthétiques ».

- avec Maurizio Ferraris, Giacomo Donis et David Web (dir.). (2001c), A taste for the Secret, Cambridge, Polity.

-. (2004a), "Questions à Jacques Derrida », dans Marc CRÉPON et Marc de Launay (dir.), La Philosophie au risque de la promesse, Paris, Bayard, p. 183-209.

- avec Giovanna BORRADORI et Jürgen HABERMAS. (2004b), Le "concept» du 11 septembre. Dialogues à New York (octobredécembre 2001), Paris, Galilée, coll. « La philosophie en effet».

-. (2006), L'animal que donc je suis, Marie-Louise MALLET (éd.), Paris, Galilée, coll. « La philosophie en effet ».

- (2008), Séminaire La bête et le souverain. Vol. I (2001-2002), Michel LisSe, Marie-Louise MALLET et Ginette Michaud (éd.), Paris, Galilée, coll. « La philosophie en effet ». 
- (2013), «Les arts de l'espace. Entretien avec Peter Brunette et David Wills ", dans Ginette MichAUD, Joana MASó et Javier BASSAS (dir.), Penser à ne pas voir. Écrits sur les arts du visible 1979-2004, Paris, La Différence, coll. « Essais », p. 15-55.

- avec Jean BAUDRILlard. (2015), Pourquoi la guerre aujourd'hui?, controverse présentée et animée par René MAJOR, Paris, Lignes.

FREUD, Sigmund. (1967 [1901]), «Déterminisme, croyance au hasard et superstition. Point de vue », dans Psychopathologie de la vie quotidienne, tr. fr. de Serge Jankélévitch, Paris, Petite bibliothèque Payot, p. 275-317.

-. (1995 [1933]), "Pourquoi la guerre?», dans OEuvres complètes, Paris, Presses universitaires de France, 1995, vol. XIX, p. 62-81.

Le Grand Robert de la langue française, Paris, Robert, <http://gr.bvdep.com/version-1/login_.asp>.

MARGEL, Serge. (2011), «Foi et savoir. L'essence du religieux, le mal radical et la question de la modernité », dans L'Avenir de la métaphysique. Lectures de Derrida, Paris, Hermann, coll. "Le Bel Aujourd'hui », p. 63-83. Une première version de ce texte a été publiée dans Marie-Louise MALLET et Ginette Michaud (dir.), Cahier de L'Herne. Derrida (2004), no 83, Paris, Éditions de L'Herne, p. 261-268.

Miller, J. Hillis. (2014), «Religio-politique de l'auto-immunité chez Jacques Derrida », tr. fr. de Brigitte Weltman-Aron, dans Danielle CoHEn-Levinas et Ginette Michaud (dir.), Appels de Jacques Derrida, Paris, Hermann, coll. " Rue de la Sorbonne », p. 261-276. Une première version de ce texte a été publiée sous le titre "Derrida Enisled» dans For Derrida (2009), New York, Fordham University Press, p. 101-133.

ReY-Flaud, Henri. (2002), Le Démenti pervers. Le refoulé et l'oublié, Paris, Aubier. 
-. (2014), Je ne comprends pas de quoi vous me parlez. Pourquoi refusons-nous parfois de reconnaître la réalité ?, Paris, Aubier.

REY, Alain (dir.) (1998), Dictionnaire historique de la langue française, Paris, Le Robert, 3 vol.

Stoller, Robert. J. (1978), La Perversion. Forme érotique de la haine, tr. fr. d'Hélène Couturier, Paris, Payot.

\section{Résumé}

Dans ce texte, il s'agit d'explorer un moment particulier du chassé-croisé conceptuel entre "méchanceté » et " perversité ». Nous examinons de quelles manières il est possible de repenser ces idées à l'aide du concept de "méchance » qu'introduit Jacques Derrida dans certains textes pour déconstruire ces notions et leurs représentations traditionnelles. Pour le philosophe, les mots contiennent en effet en eux-mêmes leur propre perversité, à savoir la possibilité, toujours présente et menaçante, de mal tomber. À partir du corpus derridien, nous nous intéressons à trois formes particulières que prend cette (mé)chance, en pragmatique du langage, en politique et en ce qui a trait au mal radical.

\section{Abstract}

In this text, we explore a peculiar moment of the conceptual crossover between "méchanceté" and "perversité." We examine how it is possible to rethink these ideas with the concept of "méchance" introduced by Jacques Derrida in some texts, especially to deconstruct these notions and their traditional representations. For the philosopher, the words contain in themselves something like their own perversity, the actual and threatening possibility of "mal tomber." From the derridean corpus, we focus on three specific forms of such a "(mé)chance", in pragmatics of language, politics and in regards to a radical otherness. 